\section{BLINKING : ITS ROLE IN PHYSICAL MEASUREMENTS}

\author{
By DR. ROBERT W. LAWSON \\ Department of Physics, University of Sheffield \\ Introductory
}

A BLINK is defined as a temporary closure of both eyes, involving movements of the upper and lower lids. The process of blinking may be carried out voluntarily; but in what follows we are primarily concerned with normal involuntary blinking. As a result of a study of the literature on this subject I have become aware of an important consequence of this lid movement which has unexpected repercussions on many phases of human activity, and which does not appear to have been recognized or appreciated hitherto. During part of the duration of each blink we undergo a complete blackout, and this has an important bearing on the accuracy attainable in a variety of physical measurements. That it has not been taken into account in the past is undoubtedly due to the involuntary nature of the act of blinking, for I find that relatively few people are aware of the blackout interval, and those who are rarely have any idea of its duration, believing it to be instantaneous. Moreover, the normal rate of blinking or the converse inter-blink period is not generally known, except in medical and kindred circles. In physics the various subjective errors of measurement have usually been considered to be taken account of adequately by the use of the personal equation. But it is now clear that the blackout factor during blinking must also enter into the personal equation, and it has to be recognized that in certain types of measurement it is, in fact, the dominant part of that equation. Before discussing this feature of the process in greater detail, it will be helpful to summarize the literature on the subject of blinking ${ }^{1}$, in so far as it is relevant to this discussion.

\section{Blinking}

(a) For the normal individual, provided that the experimental conditions remain constant, the average value of the interval between successive blinks (interblink period) is remarkably constant, though four different types of blinking have been recognized. In the first and predominant ' $J$-type', short inter-blink periods are common, the average inter-blink period being c. $2 \cdot 8$ sec. for men, and somewhat less than 4 sec. for women. In the second, 'plateau type', the average inter-blink period was found to be c. 12 sec., though most of the observations were on women subjects. For the third, 'bimodal type' (uncommon), the average period was 3.6 sec. ; and for the fourth, 'symmetrical type' (very uncommon), 5.8 sec. Dryness or humidity of the atmosphere have little effect on the rate of blinking. The value of the inter-blink period in the dark is inappreciably higher than in the light, though a sudden intense light may produce initially a few rapid blinks. The rate of blinking is more rapid when the eyes are converging on a near point than when they are focused on a distant object or moving from point to point. Attempts to stop the lid movements voluntarily lead in a short time to an almost irresistible impulse to blink.

(b) "The rate of blinking is closely related to the 'mental tension' of the subject at the time, and in all probability the movements constitute a kind of relief mechanism, whereby nervous energy, otherwise un- utilized, passes into a highly facilitated path.", When the mind is in a state of relaxation, so that the mental tension is at a low level, there is little need for relief and the rate of blinking is slow, as it is also in the process of solving a mathematical problem, when, although there is intense mental activity, the mental tension is diverted into internal channels. On the other hand, when there is mental tension with no internal or external outlet for its relief, there is an "overflow of nervous energy into facilitated paths", which manifests itself in an increased rate of blinking. As instances of this enhanced rate of blinking or diminished inter-blink period may be cited impotent rage, anxiety with inactivity, suppressed pleasure or excitement, legal cross-examination, animated conversation, and frequent changes in fixation, where blinking tends to eliminate the disturbing blurring of the retinal image during the actual movements of the eyes.

(c) The sensory blinking reflex is brought about by an irritative stimulus such as the contact of a foreign body with the lashes, cornea, or conjunctiva, or by an irritation of these parts. The reflex time of the sensory reflex seems to be of the order of 0.04 sec. and the completion of a reflex is followed by a refractory period, lasting 0.25 sec., during which a second stimulus is ineffective. The effect of currents of air is to increase the rate of blinking, and particles of dust in the eyes have the same effect. Smoking a cigarette held between the lips may reduce the inter-blink period to less than one-half the normal value, whereas in pipe-smoking less smoke enters the eyes, and $I$ have found that the inter-blink period in this case is only reduced by $16-40$ per cent.

(d) The optical blinking reflex is a completely different mechanism from the sensory, being brought about by a bright light, though it is best studied by bringing an object near to the eye without touching it. Here the reflex time is greater, being of the order of 0.1 sec. or slightly more, whereas the refractory period, during which a second stimulus is ineffective, is also longer than for the sensory reflex, amounting to $0 \cdot 5-1$ sec.

(e) The aural blinking reflex is initiated by mechanical or thermal stimulation of the ear, and is accompanied by unilateral weeping. Loud noises produce a similar effect.

$(f)$ Various estimates have been made photographically of the duration of a full blink, and these lead consistently to values of $0 \cdot 3-0.4$ sec. Ploman, Engel and Knutsson found the duration of a blink $(0.4 \mathrm{sec}$.$) to be resolvable into the following intervals :$ lowering of the upper lid, 0.05 sec.; lids closed, 0.15 sec.; raising of the upper lid, 0.20 sec. (see Duke-EIder, p. 647). From these estimates it seems fairly certain that on the evidence available the time during which the pupils of the eyes are covered by the lids cannot differ appreciably from 0.3 sec., and this value will be used in the sequence. In other words, during each full blink there is a complete blackout for a period of $\mathbf{0 . 3} \mathrm{sec}$.

(g) In a publication on eyeball reflex movement associated with blinking, Miles" writes: "It was found that synchronously with the lowering of the upper lid there was a very rapid elevation of the eyeball with a mucb slower reverse movement when the lid was lifted. This movement of the eyeball appears in both reflex and voluntary winking and seems not to depend on the condition of sleepiness. In cases where the lid reflex is small, practically the complete curve for the cornea movement is recorded. 
The size of the movement is of the order of 10 to 15 degrees on the arc of vision." "To what extent this shifting of the line of regard modifies the efficiency of vision has not been determined. Under certain lighting conditions one can easily notice the shift in the stimulus, a tailing off downward, at the instant of winking." This last statement is expressed in a somewhat different form by Macleod" : "If one notices carefully, one can observe while reading, that just at the beginning of the wink reflex it appears as if the page were suddenly jerked downwards".

In regard to this downward displacement at the moment of blinking, mentioned by Miles and Macleod, I have strong experimental evidence, to be published shortly, that it is due to another cause and not to the upward rotation of the eyeball accompanying the closing of the lids. Moreover, although an opposing motion of the eyeballs undoubtedly does occur during each phase of the act of blinking, experiments that I have conducted over a number of years have convinced me that, in general, the amount of the rotation is much less than the $10^{\circ}-15^{\circ}$ stated by Miles. Thus, in the case of my left eye, there is no perceptible rotation at all, whereas the magnitude of the rotation of my right eye is between $\frac{1}{2}^{\circ}$ and $1^{\circ}$, and I have recently met with a case in which there appears to be no rotation in either eye associated with the lid movements. Furthermore, I find that as the edge of the upper lid crosses the pupil just before and just after the blackout period, there is mobile vision, a variety of autokinetic perception, which lasts about 0.01-0.02 sec. in each case. In contrast, the type of vision that occurs during the inter-blink period may conveniently be termed static vision, and it is then that the vision is most efficient. When a single act of blinking has been completed, the eye returns to its original direction of fixation, and in my own case this occupies a time of from 0.2 to 0.25 sec. The blackout period $(0.3$ sec.) is thus followed by a period of 'mobile vision' of approximate magnitude 0.25 sec.

\section{The Blackout Indices}

The above results, taken in conjunction with the value of the inter-blink period, have some important consequences, and it will be found convenient in their discussion to introduce at this stage the terms 'blackout index' and 'modified blackout index'.

(1) The blackout index, expressed in per cent, may be defined as one hundred times the ratio between the blackout. period $(0.3$ sec.) and the inter-blink period $(2.8$ sec. for the prevalent ' $J$-type' eye-see (a) above). This means that under normal conditions a person with a $J$-type eye is 'blacked out' intermittently for 10.7 or say 11 per cent of the total time of vision.

(2) The modified blackout index, expressed in per cent, may be defined as one hundred times the ratio between the sum of the periods of blackout and mobile vision $(0.3$ sec. +0.25 sec. $=0.55 \mathrm{sec}$.$) and$ the inter-blink period. This means that under normal conditions a person with a $J$-type eye has uncertain vision, that is, is blacked out or has mobile vision, intermittently, for 19.6 or say 20 per cent of the total time of vision.

As the periods of blackout and mobile vision undoubtedly show variations from person to person, just as in the case of their inter-blink periods (section $a$ above), it is clear that the numerical data for any individual under given conditions should be separately determined in order to be able to evaluate the
TABLE 1

\begin{tabular}{|c|c|c|c|c|c|}
\hline Subject & $\begin{array}{c}\text { Inter- } \\
\text { blink } \\
\text { period } \\
\text { (sec.) }\end{array}$ & $\begin{array}{l}\text { Black- } \\
\text { out } \\
\text { period } \\
\text { (sec.) }\end{array}$ & $\begin{array}{l}\text { Mobile } \\
\text { vision } \\
\text { period } \\
\text { (sec.) }\end{array}$ & $\begin{array}{l}\text { Black- } \\
\text { out } \\
\text { index } \\
(\%)\end{array}$ & $\begin{array}{c}\text { Modifled } \\
\text { blackout } \\
\text { index } \\
(\%)\end{array}$ \\
\hline $\begin{array}{l}J \text {-type (male) } \\
\text { (female) } \\
\text { Pläteau type } \\
\text { Bimodal type } \\
\text { Symmetrical }\end{array}$ & $\begin{array}{c}2 \cdot 8 \\
c .4 \\
c .12 \\
3 \cdot 6\end{array}$ & $\begin{array}{l}0 \cdot 3 \\
0 \cdot 3 \\
0 \cdot 3 \\
0 \cdot 3\end{array}$ & $\begin{array}{l}0.25 \\
0.25 \\
0.25 \\
0.25\end{array}$ & $\begin{array}{r}11 \\
7 \cdot 5 \\
2 \cdot 5 \\
8 \cdot 3\end{array}$ & $\begin{array}{c}20 \\
14 \\
4 \cdot 6 \\
15\end{array}$ \\
\hline 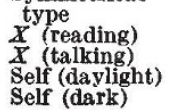 & $\begin{array}{r}5 \cdot 8 \\
1 \cdot 2 \\
0 \cdot 8 \\
13 \cdot 1 \\
13 \cdot 6\end{array}$ & $\begin{array}{l}0 \cdot 3 \\
0 \cdot 3 \\
0 \cdot 3 \\
0 \cdot 1 \\
0 \cdot 1\end{array}$ & $\begin{array}{l}0 \cdot 25 \\
0 \cdot 25 \\
0 \cdot 25 \\
0 \cdot 25 \\
0 \cdot 25\end{array}$ & $\begin{array}{l}5 \cdot 2 \\
25 \\
38 \\
0 \cdot 8 \\
0 \cdot 7\end{array}$ & $\begin{array}{l}9 \cdot 5 \\
46 \\
69 \\
2 \cdot 7 \\
2 \cdot 6\end{array}$ \\
\hline
\end{tabular}

Note.-For trained observers, the shortest normal inter-blink period so far observed is 0.6 sec., and the longest 18 sec. (woman). The lower limit of the blackout period probably lies near 0.1 sec., whereas the upper limit may readily be as large as 0.5 sec., for some subjects
screw their lids together during a blink.

blackout indices with any precision. All we can do here is to confine ourselves to the data actually available in the literature, or individually determined. In Table 1 are given the data for the main types of subject (see sections $a, f$ and $g$ ), for the rapidly blinking subject $X$, whose values I determined during reading and in animated conversation (see section $b$ ), and for myself in daylight and in the dark (see section $a$ ).

The methods used in determining my own involun. tary rate of blinking, blackout period, and mobile vision period will form the subject of a later communication. As no data on the period of mobile vision exist in the literature, the value entered in the appropriate column is that of my own eyes. From the data in the last two columns of the Table it will be seen that very wide individual variations in the blackout indices exist, and it is precisely this fact that is of great importance in physical measurements and in other spheres of human activity.

\section{Classification of Error Types due to Blinking}

Certain types of physical measurement must inevitably be in error by at least the amount indicated by the appropriate blackout index. Blackout and mobile vision errors will be present in all types of time observations, though for observers with a long inter-blink period they may be sufficiently small to be negligible, whereas for observers with a rapid rate of blinking the errors may be in excess of 50 per cent and can rarely be neglected.

In considering the subjective errors in physical me asurements due to blinking, three main types call for comment, the first of these being by far the most important.

Type A: In subjective counting experiments, where the duration of the events counted is small compared with the blackout period, and especially when the succession of events is random, the results will be in error by at least the percentage indicated by the blackout index. In certain cases the period of mobile vision may still further increase the error. The counting of scintillations in radioactivity provides an excellent illustration of this type of error, for the duration of a scintillation (see Rutherford, ref. 5, p. 545) is of the order of $10^{-4}$ sec. (blackout period 0.3 sec.), and we shall return to it later.

Type B : When the duration of the events counted or observed is of an order comparable in magnitude with the blackout or modified blackout period, the effect of blinking is an essential part of the personal equation and must be allowed for. Moreover, the error involved will be greater the more rapid the rate of blinking of the observer. 
Type $C:$ As the duration of the events observed or the time of observation is progressively increased as compared with the blackout period, the errors due to blinking likewise become progressively less important. In other words, the uncertainty of observation resulting from blinking becomes less important as the time of observation is increased, and the effect of blinking on the personal equation is diminished. This is of importance in pendulum measurements or in observations on the fall of bodies either in the air or in a viscous medium (Stokes' law). It should also be borne in mind that in measurements by the method of coincidences, individual coincidences may easily remain unobserved should they occur during the blackout period of a blink.

In my long teaching experience I have frequently been puzzled by the fact that occasionally otherwise good students seem to be incapable of getting reasonably accurate results in experiments involving the measurement of times of the order of several seconds, and have been loath to put their misfortune down to carelessness. It seems probable that this defect may be connected with the process of blinking, and I can recall individual cases of high rates of blinking in such students. I have also noticed similar discrepancies in the measurement of Young's or Fresnel's (biprism) fringes, and a feasible explanation seems to be that such observers may have a high rate of blinking, so that their vision is blacked out or mobile during a relatively high percentage of the time of their measurements with a lateral traverse micrometer eyepiece, in addition to which occasional fringes may be skipped. In microscopy, too, observers with a high modified blackout index must find it difficult to be certain of the detailed finer structure of the specimen under examination.

In various branches of industry, also, there must be many processes and measurements in which the rate of blinking may be a handicap, and consideration of the process of blinking will surely have an important part to play in matters of vocational guidance. In particular, in mining, the irritation of the eyes produced by dust and the consequent raising of the rate of blinking (see section $c$ above) may influence the safety factor, in addition to which miner's nystagmus with its accompanying mobile vision is, I understand, often associated with an enhanced rate of blinking.

\section{Application in Radioactive Measurements}

One of the most important constants in radioactivity is $Z$, the number of $\alpha$-particles emitted per second by $1 \mathrm{gm}$. of radium and by $l$ Curie of radon. Here the unit 1 Curie is used in its original sense, as the amount of radon in equilibrium with $1 \mathrm{gm}$. of radium. The value of $Z$ has been determined on many occasions and by a variety of methods. In Table 2 a summary is given of the various methods that have been used in the determination, together with the final result for each". In the words of Rutherford" : "Considering the difficulty of accurate measurement by some of the indirect methods, there is a fair accord". "There is no doubt that a direct determination of $Z$ is beset with considerable difficulties, partly connected with the source of $\alpha$-rays and partly with the efficiency of the electrical and scintillation methods of counting $\alpha$-particles." "When we consider the care taken in the measurements, it is difficult to account for the divergence between the various values of $Z$, and particularly the low values in some determinations. One of the main difficulties

\begin{tabular}{|c|c|c|c|}
\hline Method & Investigator & Date & $Z$ \\
\hline $\begin{array}{l}\text { 1. Electrical } \\
\text { counting } \\
\text { 2. Scintilla- } \\
\text { tion } \\
\text { 3. Total } \\
\text { charge } \\
\text { 4. Produc- } \\
\text { tion of } \\
\text { helium } \\
\text { 5. Life of } \\
\text { radium } \\
\text { 6. Heating } \\
\text { effect } \\
\text { 7. Volume } \\
\text { of radon }\end{array}$ & $\begin{array}{l}\text { (a) Rutherford and Geiger } \\
\text { (b) Hess and Lawson } \\
\text { (c) Geiger and Werner } \\
\text { (d) Ward, Williams and Cave } \\
\text { Geiger and Werner } \\
\text { (a) Jedrzejowski } \\
\text { (b) Braddick and Cave } \\
\text { (a) Boltwood and Rutherford } \\
\text { (b) Dewar } \\
\text { Gleditsch } \\
\text { (a) Rutherford and Robinson } \\
\text { (b) Watson and Henderson } \\
\text { Wertenstein }\end{array}$ & $\begin{array}{l}1907 \\
1918 \\
1924 \\
1929 \\
1924 \\
1927 \\
1928 \\
1911 \\
1910 \\
1919 \\
1912 \\
1928 \\
1928\end{array}$ & $\begin{array}{l}3.57 \times 10^{10} \\
3 \cdot 72 \times 10^{10} \\
3 \cdot 5 \times 10^{10} \\
3.66 \times 10^{10} \\
3 \cdot 40 \times 10^{10} \\
3 \cdot 50 \times 10^{10} \\
3 \cdot 68 \times 10^{10} \\
3.50 \times 10^{10} \\
3.69 \times 10^{10} \\
3 \cdot 55 \times 10^{10} \\
3.72 \times 10^{10} \\
3 \cdot 70 \times 10^{20} \\
3 \cdot 7 \times 10^{10}\end{array}$ \\
\hline
\end{tabular}

in these experiments lies in the choice and preparation of a suitable source of $\alpha$-rays which is free from all uncertainty and can be measured with precision."

It is of great interest to examine how the values obtained by the various subjective methods of determining $Z$ may be influenced by the rate of blinking of the observer. Without exception all the methods, directly or indirectly, involve the measurement of time intervals, usually the duration of the experiment, and so the factor of blinking will enter in some small measure into the personal equation (Type $C$ ). But the error due to the period of blackout is likely to be disturbing only in the subjective measurements of methods 1 and 2. As Rutherford and Geiger used a long-period quadrant electrometer, their blackout error can be disregarded. This also holds for the method $1(d)$, in which the particles were photographically recorded. We are thus left with methods $1(b), 1(c)$ and 2 in which the blackout and mobile vision errors may be of importance.

If the string electrometer used by Geiger and Werner in method 1 (c) was of the same type as was used by Rutherford and Geiger in their photographic recording of $\alpha$-particles, its period of deflexion would be c. 0.01 and of return 0.02 sec., both of which times are small as compared with the period of blackout during blinking $(0.3 \mathrm{sec}$.$) , so that the blackout$ error would be effective (Type $A$ ). Taking account of the blackout indices in Table 1 , this would mean that the results might readily be low by several per cent. A short-period string electrometer thus has distinct disadvantages as compared with a long-period instrument in the subjective counting of particles, a point which has not been realized hitherto.

We may now consider Geiger and Werner's scintilla. tion counting method (method 2). The question of the efficiency of scintillation counts has been the subject of much discussion ${ }^{5}$, but the above workers devised an ingenious method which would appear to guarantee one hundred per cent efficiency. In this method the same area of the screen is viewed from opposite sides by the two observers, who record their counts on a drum. If $N_{1}$ and $N_{2}$ are their respective counts, and if $C$ is the number of coincidences, then the true number of particles was shown to be $N=N_{1} N_{2} / C$. But also this method is not free from the error due to blinking, even if both observers have identical inter-blink periods and identical blackout periods, for, as already mentioned, the duration of a scintillation is c. $10^{-4}$ sec. and the period of blackout $0 \cdot 3$ sec. (Type $A$ ). Certain of their blinks would be sufficiently near to be considered practically simultaneous, especially when we remember that, after a relatively long inter-blink period, it often happens that several blinks follow in quick 
succession, so that an appreciable number of particles would not be counted by either observer. Without numerical data concerning the acts of blinking of these two workers, no correction can be applied to their estimates of $Z$, but an error of ten per cent is well within the bounds of possibility, as will be seen by reference to the fifth column of Table 1 .

In regard to scintillation counts, an abstract from some work by Chadwick" is suggestive: "When the scintillations appear on the screen at convenient intervals, it is possible for the two observers to compare their impressions of the intensity of the scintillations. It sometimes happens, particularly when dealing with the weaker scintillations, that a scintillation which appears bright to one observer is weak to the other." There seems little doubt that the weaker scintillation was seen by one of the observers during one of his periods of mobile vision (c. 0.01 and 0.25 sec.). Rutherford's remark (loc. cit.) "that the superior efficiency of an experienced observer appears to be due to greater concentration, to control of spontaneous movements of the eye, and to practice in using the ex-central portions of the retina, thereby avoiding the insensitive foveacentralis" now requires to be supplemented by the statement-- "and above all to a low value of the blackout index".

It remains to discuss method $1(b)$ in Table 2, and here numerical data on the act of blinking are available, at any rate for one of the observers (see Table 1). These workers used a longer period Elster-Geitel string electrometer for their work. The string took just under 0.2 sec. to be deflected, and the entire displaced period ${ }^{2}$ was greater than 1 sec. Since these times are appreciably greater than Lawson's blackout period $(0.1 \mathrm{sec}$.$) , it is certain, even taking$ account of his period of mobile vision $(0.25 \mathrm{sec}$.), that this observer saw at least two-thirds of a kick which occurred at the moment of a blink, so that the blackout error can be disregarded in his case. In regard to the second observer (Hess), nothing is known of his blackout period, but it is recalled that his inter-blink period was definitely shorter than that of Lawson, so that his blackout indices would be greater. A slight discrepancy in the estimates of $Z$ by these two observers might therefore be expected, and was in fact found ${ }^{8}$. Thus in the final series of observations their mean corrected values of $Z$ were :

Hess (114 determinations): $Z=3.68 \times 10^{10}$, Lawson (154 determinations) : $Z=3.75 \times 10^{10}$.

In all, about 80,000 particles were counted in these final determinations. The accuracy to be expected from this number of particles is thus of the same order as the guaranteed accuracy of secondary radium standards $(0.5$ per cent), which are used in the measurement of the sources. On the evidence available at the present time, therefore, there seems to be no valid reason for suggesting a change in the accepted value of $Z=3.70 \times 10^{10}$, for its true value is scarcely likely to be greater than $3.75 \times 10^{10}$.

\section{Further Applications of the Blackout Indices}

It is of interest to note the effect of the act of blinking in other branches of human activity. In the realm of sport it is of great importance. When we consider that a motorist who has a rapid rate of blinking may be blacked out intermittently for about 40 per cent of his driving time, or 40 per cent of the distance travelled, we can surely discern in this a primary cause of proneness to accident. In fast games like tennis or badminton, the ball or the shuttlecock will certainly be lost to sight during the $0.3 \mathrm{sec}$. of the blackout due to blinking. For people with a high rate of blinking, bowls is a much more suitable form of recreation. In flying, too, the airman does not appear to have been aware hitherto of the effect of blinking on his efficiency, either in bombing a target or in fighter combat, for in the period of his blackout or mobile vision he may have travelled a distance of the order of one hundred yards. The effect will be greater still for the pilot of a jet fighter. The blackout indices are also of great importance in cricket, for if they are high, the batsman or wicketkeeper may lose sight of the ball between the wickets, the fielder may readily miss what seemed a sure catch, and the umpire may give a wrong decision. In boxing, too, a damaged eye stimulates blinking and thus an increase in the blackout index, whereas the time constants involved in the sensory and optical blinking reflexes may be all-important to the boxer. Also in photography it is to be expected that, on the average, a group of people will have approx. imately normal time constants of blinking, and in consequence, at any moment, a definite percentage of them equal to the normal black-out index (c. 10 per cent) will be in the act of blinking. This prediction is confirmed by the examination of group photographs. Thus in two recent topical photographs of groups of 43 and 18 persons respectively, three had closed and three half-closed eyes in the first, and in the second two had closed eyes.

In conclusion, it may be mentioned that all electrical valve counting circuits, by virtue of their time constants, must suffer from the disadvantage of blackout, but as the effects are calculable, they need not necessarily involve serious error in the count. Another electrical analogy is to be found in the time base of the cathode ray oscillograph, in which the fly-back time corresponds to the blackout period in blinking. In this case, also, the error involved can readily be determined.

${ }^{1}$ In particular, Ponder, E., and Kennedy, W. P., Quart. J. Exper. Physiol., 18, 89 (1928); Blount, W. 'P., ibid., 18, 111 (1928) Luckiesh, M., and Moss, F. K., Thum. Eng., 35, 19 (1940); Hall, A., Brit. J. Ophthal., 29, 445 (1945). General references: DukeElder, W. S., "Text-Book of Ophthalmology" $1,643-647$ (Henry; 679-681 (Year Book Publishers, Inc., Chicago-Illinois, 1944).

${ }^{2}$ Miles, W. R., Amer. J. Physiol. (Proc.), 72, 239 (1925).

"Macleod's "Physiology in Modern Medicine", 278 (1941).

"Taken from "Radiations from Radioactive Substances", by Rutherford, Chadwick and Ellis, 63 (Camb. Univ. Press, 1930).

${ }^{5}$ Rutherford, Chadwick and Ellis, 544-550. See also Geiger, H. and Werner, A., $Z$. Phys., 21, 187 (1924); Hess, V. F., and Lawson, R. W., Phil. Mag., 48, 200 (1924).

'Chadwick, J., Phil. Mag., (7), 2, 1056 (1926).

' Lawson, R. W., and Hess, V. F., Wien. Ber., (IIa), 127, 465 (1918). 3. eq, V. F., and Lawson, R. W., Wien. Ber., (IIa), 127, 456 (1918).

\section{THE ECOLOGICAL APPROACH TO BOTANY}

\author{
By DR. H. GODWIN, F.R.S. \\ Botany School, Cambridge
}

$\mathrm{T}$ HE last forty yeare have witnessed the growth and establishment of the idea of ecology. Twenty years ago it was possible for hotanical professors to pronounce that they simply disbelieved in ecology, and it was a word of mystery to the general public. 1911 saw the publication of "Types of British Vegetation" by Prof. A. G. Tansley, and in 1913 the 